\title{
Contaminação bacteriana em tecla- dos de computadores utilizados em hospital universitário do nordeste do Brasil
}

\section{Bacterial contamination in computers keyboards used in university hospital of northeastern Brazil}

\author{
Anderson G Rodrigues ${ }^{1}$; Mariana AWB Viveiros ${ }^{1}$; Ivoneide MO Barroso²; Alessandra P Cavalcante ${ }^{3}$; \\ Ana MQ López ${ }^{4}$
}

\begin{abstract}
RESUMO
O avanço tecnológico permitiu a inserção dos computadores no ambiente hospitalar, sendo necessários na solicitação de exames, registro de informações dos pacientes ou para uso pessoal dos médicos. Trinta e um teclados de computadores do Hospital Universitário Professor Alberto Antunes/UFAL foram submetidos a análises microbiológicas no período de fevereiro a junho de 2011. As colônias isoladas das amostras foram analisadas morfobioquímicamente, inclusive por método automatizado (VITEK@). Entre os setores monitorados, foram detectadas as seguintes bactérias: Acinetobacter baumanii (Clínica Obstétrica), Pseudomonas stutzeri (Clínica Médica e Maternidade), P. oryzihabitans (Pediatria), Enterobacter cancerogenus (Setor de Quimioterapia), E. cloacae (UTI Neonatal), Enterococcus faecium (Enfermaria do Setor Oncológico), Sphingomonas paucimobilis (Setor Oftalmológico) e Rhizobium radiobacter (Alojamento Conjunto). Destas, P. oryzihabitans, E. cancerogenus e S. paucimobilis apresentaram resistência a antimicrobianos comumente recomendados. Portanto, os teclados de computadores hospitalares podem atuar na disseminação de patógenos, devendo fazer parte da rotina de desinfecção do ambiente hospitalar. A cobertura com película protetora e a adoção de medidas preventivas como a lavagem das mãos são de grande importância.
\end{abstract}

Palavras-chave: Computadores. Contaminação de Equipamentos. Controle de Infecções. Infecção Hospitalar.

\section{Introdução}

Infecções nosocomiais são aquelas desenvolvidas no ambiente hospitalar ou produzidas por micror- ganismos adquiridos após a admissão do paciente no mesmo, e que se manifestam durante a internação ou após a alta. ${ }^{1,2,3}$ Estudos demonstram a emergência do problema globalmente, sobretudo, quando envolve
1. Alunos do Curso de Graduação da Faculdade de Medicina da Universidade Federal de Alagoas (UFAL)

2.Médica especialista em Patologia Clínica. LACEN/AL - Laboratório Central de Saúde Pública de Alagoas

3.Farmacêutica e Bioquímica. LACEN/AL - Laboratório Central de Saúde Pública de Alagoas.

4.PhD Sc. (UK) - área de concentração Bioquímica e Biologia Molecular. Institu-to de Química e Biotecnologia da UFAL
Correspondência: Instituto de Química e Biotecnologia da UFAL Avenida Lourival Melo Mota, s/n, Tabuleiro do Martins,

Campus A. C. Simões, 57072-970 - Maceió, Alagoas, Brasil amql@qui.ufal.br.

Artigo recebido em 08/08/2011 Aprovado para publicação em 24/02/2012 
multirresistência aos anti-microbianos, estando relacionada ao aumento de morbi-mortalidade. ${ }^{4-17}$

As infecções mais comumente adquiridas em unidades de terapia intensivas (UTIs), por exemplo, incluem infecção da corrente sanguínea, pneumonia e infecção do trato urinário. ${ }^{1,12,18-23}$ As taxas de mortalidade em UTIs chegam aproximadamente a $40 \%$ e as infecções hospitalares contribuem decisivamente para este índice. Mais de $20 \%$ dos pacientes admitidos em unidades de terapia intensiva européias (UTIs) desenvolvem uma infecção adquirida nesse local. ${ }^{19} \mathrm{Em}$ 2003, Vosylius et al. ${ }^{19}$ observaram que a taxa de mortalidade de pacientes que adquiriram infecções na UTI foi significativamente mais alta em comparação com aqueles que não adquiriram estas infecções.

A contaminação das mãos é o principal fator envolvido na transmissão de infecção hospitalar e sua higienização é considerada a ação preventiva mais simples e eficaz para prevenção da disseminação microbiana nesse ambiente. ${ }^{24-29}$ Bhalla et al. ${ }^{26}$ encontraram em seus estudos dados sugestivos da provável contribuição das mãos como veículo de transmissão de patógenos nosocomiais, recomendando que as mesmas devam ser higienizadas após o contato com objetos inanimados próximos aos pacientes. No entanto, infelizmente, a falta de adesão dos trabalhadores de saúde ainda é uma realidade..$^{25,26,27}$ Este fato decorre da ausência de treinamento contínuo, pouca disponibilidade de tempo e intolerância aos sabões. ${ }^{24-29}$

$\mathrm{O}$ avanço técnico-científico permitiu um aumento dos sistemas de informatização clínica, tendo por objetivo a automação de todas as rotinas administrativas do hospital. ${ }^{2,30}$ Alguns estudos têm enfatizado a contaminação de objetos inanimados, fômites em uso nos ambientes hospitalares, assim como telefones, computadores, estetoscópios e inclusive aqueles equipamentos presentes nos leitos dos pacientes. ${ }^{31-37}$ Os teclados de computadores, por exemplo, são utilizados por mãos que podem estar contaminadas, sendo transformados em possíveis reservatórios de microrganismos. Estes, então, tor-nam-se mais uma fonte potencial de risco para a infecção hospitalar, que após manipulação dos pacientes, os trabalhadores de saúde podem transferir as causas das infecções para os mesmos, pois muitas vezes nem as luvas são retiradas antes de sua utilização.

O propósito deste trabalho foi avaliar a contaminação bacteriana de teclados de computadores utilizados em 31 diferentes setores do Hospital Universitário Prof. Alberto Antunes (HUPAA/UFAL), anali- sando o possível papel dos mesmos como fômites de infecção hospitalar, e a resistência dos microrganismos detectados frente a antimicrobianos.

\section{Material e Métodos}

\section{Amostragem/Setores}

Os setores analisados do Hospital Universitário Professor Alberto Antunes (Universidade Federal de Alagoas), HUPAA/UFAL, foram aqueles onde ocorre contato direto ou indireto com pacientes, tendo sido selecionados aleatoriamente, e constam da Tabela 1.

A coleta das amostras foi realizada nos locais citados na Tabela 1, durante o período de fevereiro a junho de 2011, utilizando swabs umedecidos em soro fisiológico estéril e friccionados sobre a superfície e laterais de teclas localizadas à esquerda, centro e direita de teclados de 31 computadores de mesa instalados em diversos setores do HUPAA/UFAL. Os swabs utilizados foram, então, introduzidos imediatamente em meio de transporte semi-sólido (Stuart) e conduzidos ao Laboratório de Microbiologia do Laboratório Central do Estado de Alagoas (LACEN-AL), em caixa de isopor contendo gel congelado, para análises microbiológicas.

\section{Processamento e Análises qualitativas}

Uma vez no laboratório, os swabs submersos em meio Stuart foram utilizados, através da técnica de esgotamento, para inoculação nos seguintes meios: CHROM-agar Orientation ${ }^{\mathrm{TM}}$, Ágar-Sangue (desfibrinado de carneiro) e Ágar-Cândida, conforme instruções do fabricante (BD). Após 48 horas de incubação a $35^{\circ} \mathrm{C} \pm 2{ }^{\circ} \mathrm{C}$, com a particu-laridade da obtenção de um ambiente a $5 \%$ de $\mathrm{CO}_{2}$ na incubação das placas de ágar-sangue, as culturas foram analisadas conforme as características das colônias obtidas. Estas foram, em seguida, replicadas em outros meios diferenciais (Agar MacConkey, Três-glicídios-ferro (TSI), e ao sistema de galerias da VITEK $®$ para organismos Gram-positivos e Gram-negativos. Além disso, os isolados foram também submetidos a testes de atividades enzimáticas específicas, como catalase e urease, e coloração de Gram, conforme descrito nos protocolos certificados para os mesmos pela legislação. As espécies bacterianas isoladas e com importância nas infecções hospitalares foram, ainda, submetidas ao Teste de Sensibilidade Antimicrobiana (TSA), mediante os métodos de disco-difusão e Etest ${ }^{\circledR}$ 


\section{Tabela 1}

Diferentes Setores do Hospital Universitário Professor “Alberto Antunes" (HUPAA) da Universidade Federal de Alagoas (UFAL), Maceió-AL, selecionados aleatoriamente para o isolamento de microrganismos em teclados de computadores, no período de fevereiro a junho/2011.

\begin{tabular}{|c|c|}
\hline Código da Amostra & Setores do hospital universitário analisados \\
\hline LAB 1 & Laboratório 1 \\
\hline LAB 2 & Laboratório 2 \\
\hline SAME & SAME \\
\hline CC 1 & Centro cirúrgico (Enfermagem) \\
\hline HID & Hospital-dia \\
\hline NEO 1 & UTI Neonatologia 1 \\
\hline OME & Central de Material Esterilizado (CME) \\
\hline PED & Pediatria $3^{\circ}$ andar \\
\hline $\mathrm{CC} 2$ & Centro cirúrgico (recepção) \\
\hline RH & Recursos Humanos \\
\hline CL.C & Clínica Cirúrgica \\
\hline NEURO & Clínica Neurológica \\
\hline $\mathbf{a M}$ & Clínica Médica \\
\hline NEO2 & UTI Neonatologia 2 \\
\hline MAT & Maternidade \\
\hline $\mathbf{C O}$ & Centro Obstétrico \\
\hline BL & Banco de leite \\
\hline $\mathrm{AC}$ & Alojamento conjunto \\
\hline UII & UTI Adulto \\
\hline LAB 3 & Laboratório 3 \\
\hline END & Endoscopia \\
\hline ADM & Administração da endoscopia \\
\hline USG & Ultrassonografia \\
\hline OFTALMO 1 & Topógrafo da oftalmologia \\
\hline OFTALMO 2 & Oftalmologia - Campo visual \\
\hline CACON 1 & Centros de Alta Complexidade em Oncologia - Quimioterapia \\
\hline CACON 2 & Centros de Alta Complexidade em Oncologia - Posto de enfermagem \\
\hline CACON3 & Centros de Alta Complexidade em Oncologia - Consultório médico \\
\hline CACON 4 & Centros de Alta Complexidade em Oncologia - Acelerador Linear \\
\hline CACON 5 & Centros de Alta Complexidade em Oncologia - Radioterapia \\
\hline FARM & Centro Cirúrgico (Farmácia) \\
\hline
\end{tabular}


(Teste de sensibilidade por gradiente de difusão), e os resultados avaliados conforme critérios do manual do "Clinical and Laboratory Standards Institute" (CLSI).

O protocolo de pesquisa foi aprovado pelo Comitê de Ética da Universidade Federal de Alagoas, atendendo à resolução 196/96.

\section{Resultados e Discussão}

Das 31 amostras obtidas de teclados de computadores dos setores selecionados aleatoriamente no HUPAA/UFAL, seis diferentes organismos de importância nosocomial foram isolados, conforme se observa na Tabela 2. Os resultados dos testes de sensibilidade a antimicrobianos de alguns dos microrganismos isolados e considerados patogênicos ao homem são apresentados na Tabela 3.

Desses isolados, Acinetobacter baumannii foi encontrado em amostras de teclados do Centro Obstétrico (CO), enquanto Pseudomonas stutzeri foi detectado nos tecla-dos da enfermaria da Clínica Médica (CM) e Maternidade (MAT). Nos teclados do Alojamento Conjunto (AC), detectou-se Rhizobium radiobacter, enquanto naqueles da UTI NEO (NEO 2) isolou-se Enterobacter cloacae. Já Enterobacter cancerogenus foi detectado nos teclados do Posto de Enfermagem do Centro de Alta Complexidade em Oncologia (CACON 2), e Enterococcus faecium nas amostras dos teclados do setor de Quimioterapia desse complexo (CACON 1). Sphingomonas paucimobilis foi isolada em amostras dos teclados do setor de Campo Visual no Ambulatório de Oftalmologia (OFTALMO 2). Binatti et al..$^{33}$ já haviam identificado a potencialidade dos teclados de computadores em albergarem e/ou disseminarem patógenos no ambiente nosocomial.

Anderson et al. ${ }^{37}$, em 2009, relataram que os teclados de computadores em três laboratórios de informática do campus de Swinburne, da Universidade de Tecnologia de Melbourne (Austrália), estavam predominantemente contaminados por Staphylococcus aureus e enterobactérias, sendo que Enterococcus faecallis colonizava todos os teclados de um único laboratório que comportava computadores com 1-3 anos de uso.

A bactéria Acinetobacter baumannii, também detectada neste estudo, é uma espécie Gram-negativa comumente isolada de amostras clínicas. No passado considerada de baixa virulência, atualmente é

\section{Tabela 2}

Microrganismos isolados dos teclados de computadores de diferentes setores do Hospital Universitário Professor "Alberto Antunes" (HUPAA) da Universidade Federal de Alagoas (UFAL), Maceió-AL (fevereiro a junho/2011)

\begin{tabular}{|c|c|c|c|c|c|c|c|c|}
\hline \multirow[b]{2}{*}{$\begin{array}{l}\text { Localização } \\
\text { Setorial* }\end{array}$} & \multicolumn{8}{|c|}{ Microrganismos Isolados } \\
\hline & $\begin{array}{c}\text { Pseudomonas } \\
\text { stutzeri }\end{array}$ & $\begin{array}{l}\text { Pseudomonas } \\
\text { oryzihabitans }\end{array}$ & $\begin{array}{l}\text { Enterobacter } \\
\text { cancerogenu }\end{array}$ & $\begin{array}{c}\text { Enterobacter } \\
\text { cloacae }\end{array}$ & $\begin{array}{l}\text { Enterococcus } \\
\text { faecium }\end{array}$ & $\begin{array}{l}\text { Sphingomonas } \\
\text { paucimobilis }\end{array}$ & $\begin{array}{l}\text { Rhizobium } \\
\text { radiobacter }\end{array}$ & $\begin{array}{c}\text { Acinetobacter } \\
\text { baumanii } \\
\text { complex }\end{array}$ \\
\hline PED & - & + & - & - & - & - & - & - \\
\hline $\mathrm{CM}$ & + & - & - & - & - & - & - & - \\
\hline NEO 2 & - & - & - & + & - & - & - & - \\
\hline MAT & + & - & - & - & - & - & - & - \\
\hline CACON 2 & - & - & + & - & - & - & - & - \\
\hline CACON 1 & - & - & - & - & + & - & - & - \\
\hline OFTALMO 2 & - & - & - & - & - & + & - & - \\
\hline $\mathrm{AC}$ & - & - & - & - & - & - & + & - \\
\hline $\mathrm{CO}$ & - & - & - & - & - & - & - & + \\
\hline
\end{tabular}

${ }^{*} \mathrm{PED}=$ Pediatria $3^{\circ}$ andar; $\mathrm{CM}=$ Clínica Médica; NEO 2= Unidade de Terapia Intensiva - Neonatologia; MAT= Maternidade; CACON 1= Centros de Alta Complexidade em Oncologia-Quimioterapia; CACON 2= Centros de Alta Complexidade em Oncologia - Posto de enfermagem; OFTALMO 2= Oftalmologia - Campo visual; $\mathrm{AC}=$ Alojamento conjunto; $\mathrm{CO}=$ Centro Obstétrico. 


\section{Tabela 3}

Sensibilidade a antimicrobianos dos microrganismos isolados dos teclados de computadores de diferentes setores do Hospital Universitário Professor "Alberto Antunes" (HUPAA) da Universidade Federal de Alagoas (UFAL), Maceió-AL (fevereiro a junho/2011).

\begin{tabular}{|c|c|c|c|c|}
\hline \multirow[b]{2}{*}{ Antimicrobianos } & \multicolumn{4}{|c|}{ nibição (mm) e Sensibilidade pelo CLSI* de Microrganismos Isolados a Antibióticos } \\
\hline & $\begin{array}{l}\text { Pseudomonas } \\
\text { oryzhiabitans }\end{array}$ & $\begin{array}{l}\text { Enterobacter } \\
\text { cancerogenus }\end{array}$ & $\begin{array}{c}\text { Enterococcus } \\
\text { faecium }\end{array}$ & $\begin{array}{l}\text { Sphingomonas } \\
\text { paucimobilis }\end{array}$ \\
\hline Benzilpenicilina & - & - & $1(\mathrm{~S})$ & - \\
\hline Ampicilina & - & $\geq 32(\mathrm{R})$ & $\leq 2(\mathrm{~S})$ & - \\
\hline Ampicilina/sulbactam & - & $16(\mathrm{I})$ & - & - \\
\hline Piperacilina/Tazobactam & $16(\mathrm{~S})$ & $\leq 4(\mathrm{~S})$ & - & $\geq 128$ (R) \\
\hline Cefalotina & - & $\geq 64(\mathrm{R})$ & - & - \\
\hline Cefoxitina & - & $\geq 64(\mathrm{R})$ & - & - \\
\hline Cefotaxima & - & $\leq 1(\mathrm{~S})$ & - & $\geq 64(R)$ \\
\hline Ceftazidima & $\leq 1(\mathrm{~S})$ & $\leq 1(\mathrm{~S})$ & - & $\leq 1(\mathrm{~S})$ \\
\hline Cefepima & $\leq 1(\mathrm{~S})$ & $\leq 1(\mathrm{~S})$ & - & $\leq 1(\mathrm{~S})$ \\
\hline Aztreonam & $\geq 64(R)$ & $\leq 1(\mathrm{~S})$ & - & $\geq 64(R)$ \\
\hline Ertapenem & - & $\leq 0,5(\mathrm{~S})$ & - & - \\
\hline Imipenem & $2(\mathrm{~S})$ & $\leq 1(\mathrm{~S})$ & - & $\geq 16(\mathrm{R})$ \\
\hline Meropenem & $1(\mathrm{~S})$ & $\leq 0,25(\mathrm{~S})$ & - & $4(S)$ \\
\hline Amicacina & $\leq 2(\mathrm{~S})$ & $\leq 2(\mathrm{~S})$ & - & $\leq 2(\mathrm{~S})$ \\
\hline Gentamicina & $\geq 16(R)$ & $\leq 1(\mathrm{~S})$ & - & $\leq 1(\mathrm{~S})$ \\
\hline Ciprofloxacina & $\leq 0,25(\mathrm{~S})$ & $\leq 0,25(\mathrm{~S})$ & $\leq 0,5(\mathrm{~S})$ & $1(\mathrm{~S})$ \\
\hline Tigeciclina & - & 2 (I) & - & - \\
\hline Moxifloxacina & - & - & $\leq 0,25(\mathrm{~S})$ & - \\
\hline Linezolid & - & - & $2(\mathrm{~S})$ & - \\
\hline Teicoplamina & - & - & $\leq 0,5(\mathrm{~S})$ & - \\
\hline Gentamicina Alto Nível (Sinergia) & - & - & $\operatorname{SIN}(S)$ & - \\
\hline Estreptomicina Alto Nível (Sinergia) & - & - & $\operatorname{SIN}(S)$ & - \\
\hline Vancomicina & - & - & $\leq 0,5(\mathrm{~S})$ & - \\
\hline
\end{tabular}

*S= Sensível; R= Resistente; I= Intermediário; SIN= Sinergia; "-"= Sem padronização pelo Manual do "Clinical and Laboratory Standards Institute" (CLSI).

reconhecida como um importante patógeno hospitalar, mais frequentemente afetando pacientes em unidades de queimados, terapias intensivas e, principalmente, sob ventilação mecânica. ${ }^{15,18} \mathrm{~A}$ infecção por esse patógeno também já foi documentada em pacientes que tenham algum tipo de imunocomprometimento, como idosos e pacientes portadores do vírus da imu- nodeficiência humana (HIV). Os sítios de infecção mais comuns são trato respiratório, sangue, urina e, menos comumente, infecções cutâneas e líquido cefalorraquidiano. Esse patógeno é capaz de sobreviver por longos períodos em superfícies secas e tem alto poder de resistência a múltiplas drogas, o que dificulta seu tratamento, piorando assim o prognóstico do paci- 
ente. Práticas em UTIs contribuem para o desenvolvimento da resistência à $A$. baumannii porque o uso de antimicrobianos pelos pacientes e em superfícies é significantemente mais alto nesses locais. ${ }^{27,32} \mathrm{~A}$ partir de 1990 as infecções por A. baumannii começaram a se disseminar rapidamente. Foi o oitavo patógeno mais frequentemente isolado de pacientes admitidos em UTIs da Espanha em 1990, com a prevalência de $3,7 \%$; dois anos depois, ele havia alcançado o terceiro lugar, com uma prevalência de $8,2 \% .^{3}$

Em 2002, Wu et al. ${ }^{18}$ também constataram que o patógeno mais frequente em infecções pulmonares associadas com a ventilação foi A. baumannii, com frequência de $27 \%$, todos de aspirado traqueal. A susceptibilidade do A. baumannii a antimicrobianos é consideravelmente diferente entre países, centros e até setores de um hospital. As diferenças de padrões de resistência em amostras enfatizam a importância de investigações locais para determinar a terapia mais adequada. A. baumannii geralmente desenvolve resistência aos aminoglicosídeos, $\beta$-lactâmicos e fluorquinolonas, e costuma ser sensível apenas aos carbapenêmicos. ${ }^{14}$ Seifert et al. ${ }^{38}$, em 1993, evidenciaram que essa espécie foi mais sensível à amicacina, ciprofloxacina e ao imipenem, e um estudo feito por Karsligil et al. ${ }^{8}$, em 2004, revelou também que a mesma mostrou-se sensível ao imipenem $(90,4 \%)$, norfloxacina $(84,5 \%)$ e ciprofloxacina $(65,4 \%)$. Por outro lado, outras pesquisas têm demonstrado sinergia ou sinergia parcial com a combinação de antimicrobianos como ampicilina/sulbactam, ticarcilina/clavulanato, piperacilina/ tazobactam e aminoglicosídeos em concentrações plasmáticas terapêuticas, embora um ou ambos agentes sejam inativos quando testados individualmente in vitro.

No Brasil, infelizmente, a resistência de bacilos Gram-negativos hospitalares à amicacina também vem aumentando em vários centros médicos, provavelmente pelo uso intenso e resistência progressiva aos aminoglicosídeos prescritos a hospedeiros imunocomprometidos. Marques et al. ${ }^{4}$, em 1997, observaram que $20 \%$ dos isolamentos de A. baumannii foram resistentes a pelo menos duas penicilinas de espectro estendido, e dois aminoglicosídeos, embora o imipenem associado a um aminoglicosídeo resultasse em sinergia total a $21 \%$ dos isolamentos altamente resistentes e sinergia parcial a $79 \%$ deles.

Pseudomonas stutzeri, por sua vez, é uma bactéria bacilar Gram-negativa, não fluorescente e denitrificante, amplamente distribuída no ambiente, mas que também tem sido eventualmente isolada como um patógeno oportunista de seres humanos, primari-amente em indivíduos debilitados. Nos últimos 15 anos, muito progresso tem sido alcançado para elucidar a taxonomia do diversificado grupo ao qual pertence, demonstrando-se a clonalidade de suas populações. Essa espécie tem recebido muita atenção devido às suas propriedades metabólicas particulares, visto serem distinguíveis de outras espécies do gênero por sua habilidade de degradar maltose e amido como únicas fontes de carbono e energia, além de ser modelo para estudos de desnitrificação; muitas cepas apresentam propriedades de transformação natural, tornando-se relevantes para o estudo de transferência de genes no ambiente. Várias cepas também são capazes de fixar o nitrogênio atmosférico, e outras participam da degradação de poluentes ou interagem com metais pesados. ${ }^{39}$ P. orzyhabitans ${ }^{20}$, por sua vez, é uma espécie não fermentante, produtora de pigmento amarelo, e que pode causar septicemia, peritonite, endoftalmite e bacteremia, sendo um patógeno oportunista de humanos e animais homeotérmicos, comumente encontrada em diversas fontes ambientais, de solo a culturas de arroz. Pode ser distinguida de outros não-fermentantes por sua reação oxidase negativa e caráter aeróbico, e pode infectar pessoas acometidas por doenças graves, incluindo aquelas submetidas à cirurgia ou com cateteres implantados ${ }^{20}$, sendo atualmente classificada como $P$. putida com base em análises de seu rDNA (ácido desoxirribonucléico ribossômico) da subunidade maior $16 \mathrm{~S} .{ }^{40} \mathrm{~A}$ estirpe dessa espécie isolada neste trabalho apresentou resistência ao monobactâmico aztreonam e ao aminoglicosídeo gentamicina (Tabela 3), ambos recomendados para o seu combate.

Em 2005, Garazzino et al. ${ }^{41}$ relataram que o sistema Nacional de Vigilância Hospitalar de Infecções nos Estados Unidos comunicara que infecções nosocomiais causadas por Enterobacter spp. estavam aumentando e isso era motivo de preocupação naquele país. Esta tendência foi confirmada em toda a Europa nos últimos anos, com Enterobacter spp. respondendo por $8 \%$ dos microrganismos isolados de unidades de terapia intensiva. Das espécies desse gênero reconhecidas, Enterobacter cloacae e Enterobacter aerogenes são rotineiramente isoladas clinicamente em humanos, enquanto as outras espécies, em sua maioria, são isoladas a partir de fontes ambientais ou de vegetais. Enterobacter cancerogenus, por sua vez, é uma das cinco novas espécies identificadas ao longo dos últimos anos. Anteriormente atribuída ao gê- 
nero Erwinia, foi transferida para o gênero Enterobacter com uma prévia sinonímia chamada Enterobacter taylorae, e após exaustivas pesquisas, sua identidade genética foi novamente reavaliada e nomeada Erwinia cancerogena e, depois, E. cancerogenus. Essa bactéria é um bacilo que fermenta lactose e tem $61 \%$ de similaridade com o DNA de E. cloacae, mas difere dele principalmente por ser ornitina negativa e Darabinose positiva. E. cancerogenus apresenta resistência natural a aminopenicilinas (amoxicilina e amoxicilina- ácido clavulânico) e/ou cefalosporinas (cefaclor, cefazolina, loracarbef, e cefoxitina $)^{41}$, o que também foi constatado no presente trabalho (Tabela 3 ). $O$ fenótipo $\beta$-lactâmico de E. cancerogenus é semelhante ao expresso por outras espécies bem conhecidas de Enterobacter, e indica a presença cromossômica de genes AmpC $\beta$-lactamases. ${ }^{41}$

Enterococcus faecium, por outro lado, é uma espécie cocóide de bactéria Gram-positiva presente na microbiota normal do trato intestinal, mas também pode ser encontrada na cavidade oral e trato vaginal, além de causar, de modo oportunista, doenças nosocomiais, como a infecção do trato urinário e de incisões operatórias, além de endocardite. Pode sobreviver por longos períodos de tempo em uma variedade de superfícies e solos, dentro de esgotos e hospitais, crescendo a temperaturas entre 10 e $45^{\circ} \mathrm{C}$ de ambientes básicos ou ácidos, isotônicos ou hipertônicos, manifestando alta resistência às drogas (vancomicina, penicilina, gentamicina, tetraciclina, eritromicina e teicoplanina), devido não só a genes cromossômicos que codificam resistência, mas também a plasmídeos e transposons conjugativos. A disseminação da doença ocorre entre os pacientes hospitalizados graças à veiculação pelas mãos ou instrumentos médicos contaminados. O uso de antibióticos também pode diminuir a prevalência de microrganismos intestinais que competem com essa bactéria que coloniza muitos órgãos do corpo, incluindo o próprio trato gastrointestinal e a pele. Além disso, a proteína de superfície de enterococos $(E s p)$, a qual permite a formação de biofilme, leva à colonização de cateteres utilizados em hospitais, causando infecções do sangue e do trato urinário, contribuindo para a virulência dessa espécie. O gene $E s p$ é normalmente presente em isolados clínicos, porém não em cepas que colonizam o intestino naturalmente. A regulação do gene Esp permite a E. faecium, portanto, mudar sua resposta ao entrar em um hospedeiro. Por exemplo, a expressão desse gene aumenta com a elevação da temperatura e/ou com uma mudança para condição anaeróbica. Fatores de virulência incluem ainda a substância de agregação (permite a adesão da bactéria às células-alvo e facilita a transferência de material genético), além da citosolina (proteína citossólica que lisa os eritrócitos) e da gelatinase (hidrolisa peptídeos). A presença de fatores de virulência difere entre as cepas e, geralmente, estes são específicos para o hospedeiro colonizado. Ao contrário de Enterococcus faecalis, E. faecium pode produzir reação $\alpha$-hemolítica no Ágar-sangue e é incapaz de utilizar ácido pirúvico como fonte energética. ${ }^{36,42}$

O gênero Sphingomonas - assim denominado em 1990 por Yabuchhi et al. ${ }^{43}$ devido à presença de esfingoglicolipídeo (GSL) contendo ácido glucurônico na superfície de suas células, dos tipos GSL-1 ou GSL$4,{ }^{44}$ apresenta metabolismo estritamente aeróbico com algumas espécies produtoras de bacterioclorofila "a". HEUNG et al., em 2006, relataram que os GSL têm papel crucial na regulação de processos patogênicos em células humanas. ${ }^{44}$ A coloração das colônias é predominantemente amarela, com variação de tonalidade de espécie para espécie e de acordo com os meios de cultura utilizados e tempo de incubação, devido a expressão do carotenóide nostoxantina. Uma de suas espécies anteriormente denominada Pseudomonas paucimobilis (sinonímia de Flavobacterium devorans) foi reclassificada como $S$. paucimobilis, a qual pertence à sub-classe $\alpha$-proteobacteria, sendo Gramnegativas não formadora de esporos, com células bacilares ou cocobacilares retas ou curvas, reprodução por fissão binária assimétrica e, em geral, sem flagelos. S. paucimobilis é uma espécie encontrada em solo e águas, sendo uma causa rara de infecções associadas à saúde humana, mas já foi relatada como causadora de surtos. ${ }^{21,22}$ Em novembro de 2007, por exemplo, o Departamento de Epidemiologia e Controle de Infecção Hospitalar do "The Johns Hopkins Hospital" iniciou uma investigação de surtos após ser notificado pelo laboratório de microbiologia do hospital sobre o crescimento de $S$. paucimobilis em hemoculturas de vários pacientes ao longo de um período duas semanas. Um surto em recém-nascidos sob ventilação mecânica esteve associado a sensores de temperatura contaminados. ${ }^{22}$ Também já foi associada à bacteremia entre pacientes imunodeprimidos em hematologia e oncologia, e descrita como contaminante de sistemas de água hospitalar. Nesse mesmo estudo, menciona-se que as cepas isoladas foram sensíveis ao antibiótico imipenem ou a um aminoglicosídeo associado a uma cefalosporina de terceira geração. ${ }^{21}$ Já 
no presente trabalho, a cepa de S. paucimobilis isolada foi resistente ao imipenem, ao monobactâmico aztreonam e ao $\beta$-lactâmico piperacilina/tazobactam (Tabela 3).

Por outro lado, Rhizobium radiobacter é uma bactéria Gram-negativa frequentemente encontrada no solo, e oportunista incomum como patógeno. Tem sido associada a dispositivos intravasculares em hospedeiros imunocomprometidos. Em 2008, relatou-se o primeiro caso de um paciente que desenvolveu bacteremia causada por $R$. radiobacter devido à exposição ao solo após a implantação de cateter venoso central, para o acesso a quimioterapia contra mieloma múltiplo. ${ }^{23}$

Portanto, dois fatores desempenham um papel na interação entre qualquer fômite, tal como teclados de computadores, e os pacientes, isto é, a sobrevivência de um microrganismo em particular numa superfície determinada ${ }^{45}$ e o fato de que vários vetores, tais como os servidores da área da saúde num hospital, além de ácaros, formigas e alguns outros pequenos insetos, podem transferir esses microrganismos de um ambiente para outro. Isso varia de um microrganismo para outro, de uma superfície específica para outra, da concentração do inóculo e de sua forma de reprodução. No caso das bactérias isoladas neste trabalho e da superfície plástica de computadores, onde existem ranhuras contendo resíduos de matéria orgânica nutritiva proveniente das mãos do manipulador, a sobrevivência é de minutos a poucos dias, dependendo do grau e frequência da assepsia. Já a possibilidade de um paciente se contaminar diretamente é muito pequena, mas se um funcionário do hospital atuar como vetor, a chance de reprodução e contaminação provavelmente aumenta muito. Em 2006, Rutala et al. ${ }^{34}$ verificaram a eficácia, após exposição por 60 minutos, de seis diferentes desinfetantes (três contendo amônio quarternário e três contendo respectivamente cloro, álcool e fenol), contra microrganismos previamente isolados em 25 teclados de computadores das clínicas da Universidade da Carolina do Norte, nos Estados Unidos. Os patógenos incluíam espécies de
Micrococcus, Bacillus, Streptococcus, Staphylococcus, Enterococcus e Salmonella, sendo muitas das espécies resistentes a antibióticos específicos, como oxacilina (Staphylococcus) e vancomicina (Enterococcus). Da mesma forma, Fukada et al. ${ }^{36}$, em 2008, avaliaram o grau de contaminação e sensibilidade a desinfetantes, de teclados de computadores de salas de cirurgia, verificando se os anestesistas lavavam as mãos antes ou depois da anestesia. Constataram a presença de contaminantes e associaram isso também ao uso dos computadores por anestesistas que não lavavam as mãos adequadamente antes da cirurgia (17\%), embora 64-69\% dos anestesistas praticavam a higienização das mãos após aplicarem a anestesia ou previamente ao almoço. A limpeza dos teclados com etil-álcool efetivamente reduziu a contaminação dos mesmos nas salas de cirurgia.

Dessa forma, considerando todas as possibilidades dos organismos isolados neste trabalho de manifestarem resistência a antibióticos, não apenas após exposição indiscriminada aos mesmos ou conjugação com outros microrganismos resistentes, favorecendo a infecção em pacientes imunocomprometidos, é de suma importância que os componentes da comissão de infecção hospitalar do HUPAA/UFAL incluam entre os materiais vistoriados em suas inspeções também os teclados de computadores. Isso deve ser associado à orientação dos trabalhadores das unidades de saúde sobre quais protoco-los seguir para evitar futuros casos de infecções nosocomiais. As alas do hospital devem permanecer abertas somente após o cumprimento das exigências de higienização, evitando a necessidade de solicitação de inspetores da Vigilância Sanitária para avaliação das instalações do Hospital em questão.

\section{Agradecimentos}

Laboratório Central de Saúde Pública de Alagoas (LACEN/AL) e Instituto de Química e Biotecnologia da Universidade Federal de Alagoas (IQB/UFAL).

\section{ABSTRACT}

The technological advance allows the insertion of computers in the hospital environment, being needed to the execution of tests, record the patient information or doctor's personal use. Thirtyone samples of computer keyboards from the Hospital "Professor Alberto Antunes", at the Federal University of Alagoas, Brazil (UFAL), were subjected to microbiological analysis in the period from February to June 2011. The colonies that were isolated were subsequently morphologically and biochemically analyzed, including by 
automated method (VITEK®). Among the sectors monitored, the following bacteria were detected: Acinetobacter baumannii (Obstetrical Clinic), Pseudomonas stutzeri (General Medicine and Maternity), P. oryzihabitans (Pediatrics), Enterobacter cancerogenus (Chemotherapy Sector), E. cloacae (Neonatal Intensive Care Unit), Enterococcus faecium (Oncology Nursing Sector), Sphingomonas paucimobilis (Ophtalmology Sector) and Rhizobium radiobacter (rooming). From these, P. oryzihabitans, E. cancerogenus and $S$. paucimobilis showed resistance to antibiotics commonly recommended. Therefore, the hospital computer keyboards can play in the spread of pathogens and should be part of the routine disinfection of the hospital. Cover with protective film and the adoption of preventive measures such as hand washing are important.

Keywords: Computer. Equipment Contamination. Infection Control. Cross Infection.

\section{Referências}

1. Fridkin SK, Welbel SF, Weinstein RA. Magnitude and prevention of nosocomial infections in the intensive care unit. Infect Dis Clin North Am 1997; 11: 479-96.

2. Harris AD. How important is the environment in the emergence of nosocomial antimicrobial-resistant bacteria? Oxford J Clin Infect Dis 2008; 46:686-8.

3. Vaqué J, Rosselló J, Trilla A, Monge V, Garcia Caballero J, Arribas JL, et al. Nosocomial infections in Spain: results of five nationwide serial prevalence surveys (EPINE Project, 1990 to 1994). Nosocomial infections prevalence study in Spain. Infect Control Hosp Epidemiol. 1996; 17:293-7.

4. Marques MB, Brookings ES, Moser SA, Sonke PB, Waites $\mathrm{KB}$. Comparative in vitro antimicrobial susceptibilities of nosocomial isolates of Acinetobacter baumannii and synergistic activities of nine antimicrobial combinations. Antimicrob Agents Chemother. 1997; 41: 881-5.

5. Collins SM, Hacek DM, Degen LA, Wright MO, Noskin GA, Peterson LR. Contamination of the clinical microbiology laboratory with vancomycin-resistant enterococci and multidrugresistant enterobacteriaceae: implications for hospital and laboratory workers. J Clin Microbiol. 2001; 39: 3772-4.

6. Bhalla A, Pultz NJ , Ray AJ, Hoyen CK, Eckstein EC, Donskey CJ. Antianaerobic antibiotic therapy promotes overgrowth of antibiotic? resistant, gram? negative bacilli and vancomycin? resistant enterococci in the stool of colonized patients. Infect Control Hosp Epidemiol. 2003; 24: 644-9.

7. Cesur S, Çokça F. Nasal carriage of methicillin-resistant staphylococcus aureus among hospital staff and outpatients. Infect Control Hosp Epidemiol. 2004; 25: 169-71.

8. Karsligil T, Balci I, Zer Y. Antibacterial sensitivity of acinetobacter strains isolated from nosocomial infections. J Int Med Res. 2004; 32: 436-41.

9. Lepelletier D, Perron S, Huguenin H, Picard M, Berner P, Caillon J, et al. Which Strategies Follow From the Surveillance of Multidrug-Resistant Bacteria to Strengthen the Control of Their Spread? A French Experience. Infect Control Hosp Epidemiol. 2004; 25: 162-4.

10. Loeb M, McGeer A, Simor A. Walter, SD, Bradley S, Low DE, et al. Facility-Level Correlates of Antimicrobial Use in Nursing Homes. Infect Control Hosp Epidemiol. 2004; 25: 173-6.

11. Marc-Oliver BSW, Joan N. Hebden RN, Harris AD, Shanholtz $\mathrm{CB}$, Standiford HC, Furuno JP, et al. Aggressive control measures for resistant acinetobacter baumannii and the impact on acquisition of methicillin-resistant staphylococcus aureus and vancomycin-resistant enterococcus in a medical intensive care unit. Infect Control Hosp Epidemiol. 2004; 25: 167-8.
12. Martins ST, Moreira M, Furtado GHC, et al. Application of control measures for infections caused by multiresistant Gram- negative bacteria in intensive care unit patients. Mem Inst Oswaldo Cruz. 2004; 99: 331-4.

13. Rosario-Rosado RV, René AA, Jones B. Descriptive analysis of patients with community-onset and hospital-onset methicillin-resistant staphylococcus aureus infections. Infect Control Hosp Epidemiol. 2004; 25: 171-3.

14. Poirel L, Nordmann P. Carbapenem resistance in acinetobacter baumannii: mechanisms and epidemilogy. Clin Microbiol Infect. 2006; 12:826-36.

15. Wisplinghoff $H$, Schimitt $R$, Wöhrmann A, Stefanik D, Seifert $H$. Resistance to disinfectants in epidemiologically defined clinical isolates of Acinetobacter baumannii. J Hosp Infect. 2007; 66:174-81.

16. Chen CY, Hansen KS, Hansen LK. Rhizobium radiobacter as an opportunistic pathogen in central venous catheter-associated bloodstream infection: case report and review. J Hosp Infect. 2008; 68(3):203-7.

17. Po-Liang Lu; LK Siu; Tun-Chieh Chen; Ling Ma; Wen-Gin Chiang; Yen-Hsu Chen, et al. Methicillin-resistant staphylococcus aureus and acinetobacter baumannii on computer interface surfaces of hospital wards and association with clinical isolates. BMC Infect Dis. 2009; 9: 1-7.

18. Wu CL, Yang DI, Wang NY, Kuo HT, Chen PZ. Quantitative culture of endotracheal aspirates in the diagnosis of ventilator-associated pneumonia in patients with treatment. Chest. 2002; 122:662-8.

19. Vosylius S, Spylaite J, Ivaskevivius J. Intensive care unit acquired infection: a prevalence and impact on morbidity and mortality. Acta Anaesthesiol Scand. 47 2003; 1132-7.

20. Lejbkowicz F, Belavsky L, Kudinsky R, Gery R. Bacteraemia and sinusitis due to flavimonas orzyhabitans infection. Scand J Infect Dis. 2003; 35: 411-14.

21. Kilic A, Senes Z, Kurekci AE, Aydogan H, Senser H, Kismet E, et al. Nosocomial outbreak of sphingomonas paucimobilis bacteremia in a hemato/oncology unit. Jpn J Infect Dis. 2007; 60: 394-6.

22. Maragakis LL, Chaiwarith R, Srinivasan A, Torriani FJ, Avdic E, Lee A, et al. Sphingomonas paucimobilis bloodstream infections associated with contaminated intravenous fentanyl. Emerg Infect Dis. 2009; 15: 12-18.

23. Sood S, Nerurkar V, Malvnkar S. Catheter associated bloodstream infection caused by $\mathrm{R}$. radiobacter. Indian $\mathrm{J}$ Med Microbiol. 2010; 28:62-4. 
24. Lucet J, Rigaud M, et al. Hand contamination before and after different hand hygiene techniques: a randomized clinical trial. J Hosp Infect. 2002; 50: 276-80.

25. Barboza DB, Soler ZASGS. Afastamentos do trabalho na enfermagem: ocorrências com trabalhadores de um hospital de ensino. Rev Latinoam Enferm. 2003; 11: 177-83.

26. Bhalla A, Pultz NJ, Gries DM, Ray AJ, Eckstein EC, Aron DC, Donskey CJ. Acquisition of nosocomial pathogens on hands after contact with environmental surfaces near hospitalized patients. Infect Control Hosp Epidemiol. 2004; 25: 164-7.

27. Martins PP, Costa CRM, Tórtora JCO. Contaminação microbiana nas mãos de pessoas com diferentes atividades profissionais. J Bras Med. 2005; 88:10-15.

28. Martins PP, Costa CRM, Tórtora JCO. Avaliação microbiológica de processos para higienização das mãos em ambiente não-hospitalar. J Bras Med. 2005; 89: 12-17.

29. Santos AAM. Higienização das mãos no controle das infecções em serviços de saúde. ANVISA. Disponível em: <http://www.anvisa.gov.br>. Acessado em 03/05/10.

30. Ilha OJO. Registro clínico computadorizado no hospital. Rev Inf. 1993; 1: 5-8.

31. Neely NA, Maley MP. Teclado de computador contaminado e microbiota sobrevivente. Am J Infect Control. 2001; 29:131-2.

32. Person OC, Lopes AF, Nardi JC, Aringa ARD, Tanaka II. Avaliação da flora bacteriana dos fones de ouvido de telefones públicos e hospitalares de Marília. Arq Med ABC. 2005; 30: 34-8.

33. Binatti VB, Costa CRM, Tórtora JCO. Patógenos hospitalares resistentes em teclados de computadores. J Bras Med. 2006; 90: 28-37.

34. Rutala WA, White MS, Gergen MF, Weber DJ. Bacterial contamination of keyboards: efficacy and functional impact of disinfectants. Infect Control Hosp Epidemiol. 2006; 27:3727.

35. Dogan M, Feyzioglu B, Ozdemir M, Baysal B. Investigation of microbial colonization of computer keyboards used inside and outside hospital environments. Mikrobiyol Bul. 2008; 42: 331-6.
36. Fukada T, Iwakiri H, Ozaki M. Anaesthetists' role in computer keyboard contam-ination in an operating room. J Hosp Infect. 2008; 70:148-53.

37. Anderson G, Palombo EA. Microbial contamination of computer keyboards in a university setting. Am J Infect Control. 2009; 37:507-9.

38. Seifert H, Baginski R, Schulze A, Pulverer G. Antimicrobial susceptibility of Acinetobacter species. Antimicrob Agents Chemother. 1993; 37: 750-3.

39. Lalucat J, Bennasara A, Bosch R, García-Valdés E, Palleroni NJ. Biology of Pseudomonas stutzeri. Microbiol Mol Biol Rev. 2006; 70: 510-47.

40. Anzai Y, Kim H, Park JY, Wakabayashi H, Oyaizu H. Phylogenetic affiliation of the pseudomonads based on $16 \mathrm{~S}$ rRNA sequence. Int J Syst Evol Microbiol. 2000; 50: 1563-89.

41. Garazzino S, Aprato A, Maiello A, Massé A, Biasibetti A, De Rosa FG, et al. Osteomyelitis caused by enterobacter cancerogenus infection following a traumatic injury: case report and review of the literature. J Clin Microbiol. 2005; 43: 1459-61.

42. Van Wamel WJB, Hendrickx APA, Bonten MJM. Growth condition-dependent esp expression by enterococcus faecium affects initial adherence and biofilm formation. Infect Immun. 2007; 75:2:924-32.

43. Yabuuchi E, Yano I, Oyaizu H, Hashimoto Y, Ezaki T, Yamamoto $\mathrm{H}$. Proposals of Sphingomonas paucimobilis gen. nov. and comb. nov., Sphingomonas para-paucimobilis sp. nov., Sphingomonas yanoikuyae sp. nov., Sphingomonas adhaesiva sp. nov., Sphingomonas capsulata comb. nov., and two genospecies of the genus Sphingomonas. Microbiol Immunol. 1990; 34:99-119.

44. Heung LJ, Luberto C, Del Poeta M. Role of Sphingolipids in Microbial Patho-genesis. Infect Immun. 2006; 74: 28-39.

45. Inocente FR, Gomes FR, Raiguier IM. Incidência de Staplylococcus aureus e de bactérias da família Enterobacteriaceae em cédulas de $R \$ 1,00, R \$ 5,00, R \$ 10,00$ e $R \$$ 50,00. Rev Estud Biol. 2004; 26: 21-6. 